\title{
Impact of the Solvent Quality on the Local Dynamics of Soft and Swollen Polymer Nanoparticles Functionalized with Polymer Chains
}

\author{
Young-Gon Kim and Héloïse Thérien-Aubin*
}

Cite This: Macromolecules 2020, 53, 7561-7569

Read Online

ABSTRACT: Grafting polymer chains on the surface of nanoparticles (NPs) is a strategy used to control the interaction between the NPs and their environment. The fate of the resulting particles in a given environment is strongly influenced by the solvent-polymer interaction. The solvent quality affects the behavior, conformation, and dynamics of the grafted polymer chains. However, when this polymer grafting strategy is used to functionalized polymer particles, the influence of solvent quality becomes even more complex; when the grafted polymer chains and the polymer nanoparticles are tethered together, the effect of the solvent quality on the behavior and dynamics of the system depends on the solvent interaction with both polymer components. To explore the relationship between the

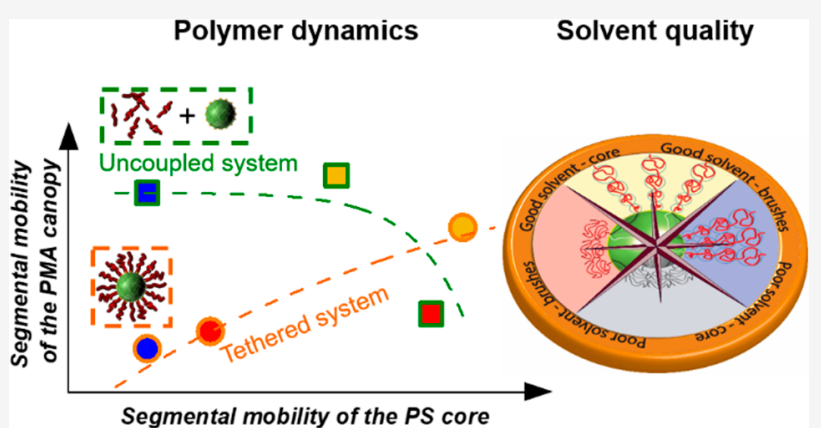
solvent quality and the dynamics of polymer-functionalized soft polymer NPs, we designed a system based on cross-linked polystyrene (PS) NPs grafted with a canopy of poly(methyl acrylate) (PMA). PS and PMA, two immiscible polymers, can be selectively solvated by using binary mixtures of solvents. NMR spectroscopy was used to address the effect of those selective solvents on the local mobility of the PS-PMA core-canopy NPs and revealed an interplay between the local mobility of the core and the local mobility of the canopy. A selective reduction of the solvent quality for the PMA canopy resulted in the expected reduction of the local mobility of the PMA chains, but also in the slower dynamics of the PS core. Similarly, a selective reduction of the solvent quality for the PS core resulted in a slower dynamics for both the PS core and the PMA canopy.

\section{INTRODUCTION}

The grafting of end-tethered polymer chains on a surface is an excellent strategy to control the properties of interfaces such as the surface of nanoparticles (NPs) in suspension. ${ }^{1,2}$ Nanoparticles functionalized with a canopy of end-tethered polymer chains have been used in a variety of applications, such as drug delivery, adhesives, or coatings. ${ }^{3-6}$ Using a canopy of endtethered polymer chains to tune the interactions between the nanoparticles and their environment is a strategy widely employed to control the dispersibility of nanoparticles both in suspension and in polymer matrixes for the formation of nanocomposites with specific properties. ${ }^{7,8}$ The properties of the resulting functionalized NPs are affected by the chemical composition of the polymer canopy, its architecture, and its interaction with the environment. The dynamics of the tethered chains is a piece of crucial information to understand the behavior of such systems. The chain dynamics in polymerfunctionalized NPs has been studied for a variety of rigid nanoparticles core ${ }^{9}$ such as gold, ${ }^{10}$ silica, ${ }^{11}$ or carbon black. ${ }^{12}$ However, new polymer-grafted systems used in drug delivery or tissue engineering rely on softer NP cores like polymer micelles or swollen nanogels. ${ }^{13,14}$ When the polymer chains are tethered to such soft and deformable NP cores, tuning the properties of the polymer canopy to optimize the behavior of the functionalized nanoparticles represents a particular challenge since both the canopy of end-tethered chains and the core of the nanoparticles are soft, deformable, and strongly affected by the conditions of the surrounding environment such as the solvent quality. ${ }^{15}$

The behavior of polymer-grafted NPs is tunable through the control of the polymer chain conformation, which is influenced by the architecture of the polymer canopy defined by the grafting density and the chain length of the end-tethered polymer chains. For example, polystyrene-grafted silica nanoparticles in a polystyrene matrix displayed tunable aggregation states, forming short strings, sheets, or clusters, by controlling the molecular weight and grafting density of the end-grafted polystyrene chains. ${ }^{7}$ This behavior was attributed to the conformation of the end-tethered chains and the architecture of the polymer-grafted nanoparticles. The conformational changes experienced by the end-tethered chains are related to the local concentration of monomer units. Chains tethered

Received: February 13, 2020

Revised: July 28, 2020

Published: August 21, 2020 
to a substrate at a high grafting density will experience a stronger steric hindrance from the neighboring chains leading to a larger reduction of conformational entropy than chains tethered at a lower grafting density. Similarly, when chains are tethered to a spherical substrate, like a nanoparticle, the local monomer concentration will decrease radially from the surface of the core nanoparticle to the interface between the surface of the polymer canopy and the surrounding environment, and the steric hindrance and the reduction of conformational entropy will decrease analogously. Consequently, the chains grafted to a NP experience a distribution of confinement states. ${ }^{16}$ The variation in the extent of confinement results in different interactions between the polymer-functionalized NPs themselves and between the polymer-functionalized NPs and their environment. $^{16,17}$

The study of the polymer chains dynamics and conformation is used to understand the effects of architecture and solvent quality on the behavior of functionalized NPs. ${ }^{11,18-20}$ Different techniques such as dielectric spectroscopy, neutron spin-echo, or neutron backscattering spectroscopy ${ }^{21-24}$ are probing different time scale and thus different aspects of the polymer dynamics. ${ }^{25}$ High-field NMR relaxometry compares advantageously to other techniques. It can be used to investigate fast relaxation processes associated with the motions, vibrations, and rotation of side groups and it probes the subsegmental and Rouse dynamics of the polymer chains. ${ }^{25-32}$ NMR spectroscopy provides results in keeping with other techniques like dielectric spectroscopy or quasielastic neutron scattering ${ }^{33}$ with the added benefit of allowing for the analysis of the local dynamics of specific chemical groups without the need for labeling. Both NMR spectroscopy and more specifically NMR relaxometry have become more popular to characterize polymer functionalized nanoparticles both in the solid-state and in suspension. ${ }^{34-37}$

The behavior of the functionalized NPs is not only influenced by the architecture of the polymer canopy, but also by the solvent quality. The chain dynamics and the conformation of end-grafted chains are also affected by the polymer-solvent interaction, ${ }^{20,38}$ and changes in the solvent quality have been used to control the properties of polymerfunctionalized NPs. ${ }^{39,40}$ When end-tethered polymer chains are in a good solvent (Flory-Huggins interaction parameter, $\left.\chi_{12}<0.5\right)$, both enthalpic attraction between chain segments and solvent molecules and entropic repulsion between chain segments occur in the polymer canopy ${ }^{41}$ and results in an extended chain conformation of the end-grafted polymer chains. Consequently, the swelling of the polymer canopy on the NPs in the suspension provides colloidal stability through steric repulsions. However, when the quality of the solvent decreases, the conformation of the end-grafted polymer chains can undergo a transition from a stretched regime to a more collapsed regime and can be accompanied by the formation of nonuniform polymer canopy ${ }^{42,43}$ or lead to the self-assembly of polymer chain grafted NPs into various mesostructures. ${ }^{44,45}$

When dealing with swollen polymer NPs, the influence of the solvent quality for the core NPs on the behavior of the suspension is also critical. Soft NPs are deformable in comparison to the inorganic hard NPs and can respond to external stimuli such as temperature, $\mathrm{pH}$, and solvent quality. Under good solvency conditions, the polymer NPs are swollen with solvent and are soft and deformable, but when the solvent quality decreases, deswelling is observed and results in a variation in the rheological behavior, optical properties, interfacial behavior, aggregation, or self-assembly of the NPs in suspension. ${ }^{46-50}$ For example, when the quality of the solvent of aqueous suspensions of poly- $\mathrm{N}$-isopropylacrylamide (PNIPAM) soft microgels is decreased either by increasing the temperature or by the addition of a cononsolvent, the volume occupied by the particles in suspension decreases leading to the obtention of collapsed microgel, a reduced segmental chain dynamics, and a diminution in the viscosity of the suspension. ${ }^{51,52}$

In the case of polymer NPs functionalized with end-tethered polymer chains, the role of solvent quality is complex, but understanding the role of solvent quality on the dynamics, conformation, colloidal stability, and properties is crucial in the design of new functional and responsive nanomaterials used for example in drug release application. ${ }^{53}$ The contribution of the solvent quality on the swelling and dynamics of both the core and the end-tethered layers needs to be studied in more detail. The interplay between the dynamics of the shell and of the core NP needs to be better understood. ${ }^{54,55}$ Here, to understand the effect of solvent quality on the dynamics of swollen polymer NPs functionalized with a canopy of polymer chains, we functionalized cross-linked polystyrene (PS) NPs with a canopy of poly(methyl acrylate) (PMA). PS and PMA are two immiscible polymers that can be selectively solvated using binary mixtures of solvent (Figure 1).

\section{EXPERIMENTAL SECTION}

Synthesis of Polystyrene Nanoparticles Functionalized with a Canopy of Poly(methyl acrylate) Chains (PS-PMA NPs). The synthesis of the PS-PMA NPs proceeded in a multistep process (Figure 1). First, the PS cores were prepared using miniemulsion polymerization. When the monomer conversion reached $90 \%$, a new monomer mixture containing an ARTP inimer containing a disulfide bond was slowly fed to the system to create a thin shell of ATRPinitiator containing polymer. After the thorough washing of the nanoparticles, the PS cores functionalized with the ATRP initiator were redispersed in anisole and used to initiate the polymerization of methyl acrylate. After different polymerization times, the polymerization was quenched, and the resulting polymer functionalized PSPMA nanoparticles were precipitated, followed by redispersion in DCM. The detailed procedure is available in the Supporting Information.

Characterizations. The PS NPs, PS-PMA NPs, and PMA free chain were dispersed in deuterated solvent mixtures at a concentration of $16.7 \mathrm{mg} \mathrm{mL}^{-1}$. The relaxation experiments were performed on NMR AVANCE spectrometers (Bruker), working at a nominal frequency of $300.13 \mathrm{MHz}$. The spin-spin relaxation time constants $\left(T_{2}\right)$ were measured using the Carr-Purcell-Meiboom-Gill (CPMG) pulse sequence. The protons in the aromatic ring of PS (7.1 to $6.6 \mathrm{ppm})$ and in the methoxy group (3.7 ppm) were chosen for the analysis.

\section{RESULTS AND DISCUSSION}

PMA grafted PS soft NPs were prepared via miniemulsion polymerization, followed by surface-initiated atom transfer radical polymerization. ${ }^{56-58}$ In the present study, PS NPs were sparsely cross-linked ( $0.5 \mathrm{~mol} \%$ of divinylbenzene) and functionalized with PMA chains of increasing lengths, and the five different molecular weight varied from 4 to $52 \mathrm{kDa}$. The grafting density of the PMA chains was $0.20 \pm 0.02$ chains $\mathrm{nm}^{-2}$ when the NPs were dispersed in dichloromethane (DCM). The NPs were dispersed in solvent mixtures of DCM with either cyclohexane or acetone to selectively control the solvent quality. The local dynamics of the PS core and of the 
(a)

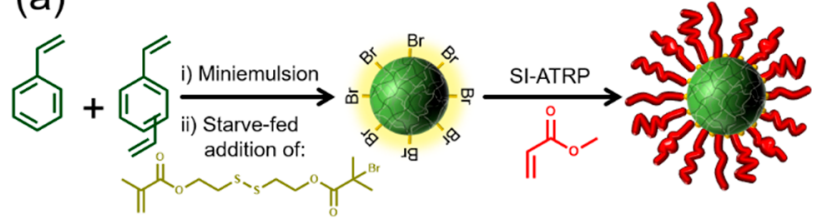

(b)

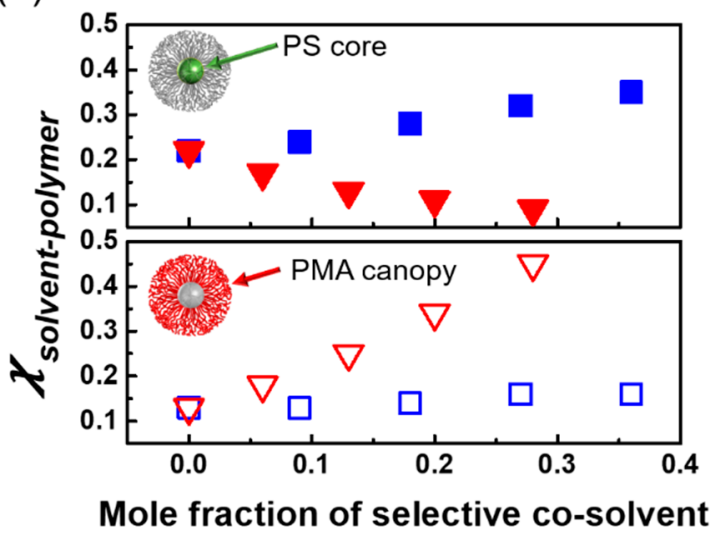

(c)

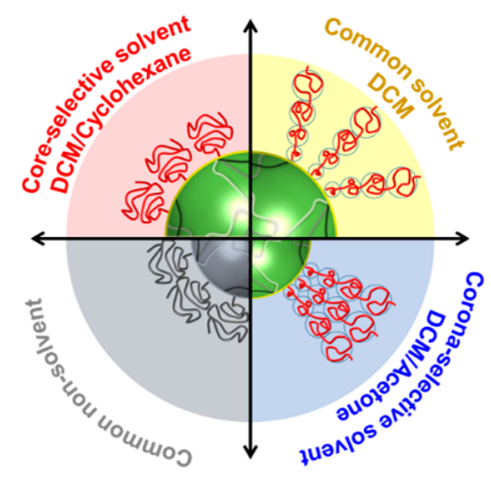

Figure 1. (a) Synthesis of PS-PMA NPs. (b) Polymer-solvent Flory-Huggins interaction parameter $\left(\chi_{\text {polymer-solvent }}\right)$ for PS (upper panel, closed symbol) and PMA (lower panel, open symbol) with solvent mixtures of DCM and cosolvents, cyclohexane (red), and acetone (blue). The $\chi_{\text {polymer-solvent }}$ were calculated from the Hansen solubility parameters of the polymers and solvents (Tables S2 and S3) using eq S1. (c) The expected effect of the relative solvent quality on the swelling of the PS-PMA NPs.

PMA canopy were addressed independently using the spinspin relaxation time obtained by NMR spectroscopy.

NMR relaxometry is especially ideal for interpreting the dynamics of soft NPs with a surface functionalization of endtethered polymer chains because the measurements can resolve the relaxation of specific chemical entities such as the polymer chains anchored on the surface, or the cross-linked polymer network in the core NPs. ${ }^{55,59,60}$ The spin-spin relaxation characterized by the relaxation time $T_{2}$ is the result of the interactions between the rotating spins in the system without energy exchange, ${ }^{61}$ and it is used to study the effect of temperature, molecular weight, cross-linking density or entanglement on the molecular mobility. The $T_{2}$ is an ideal marker of the local dynamics in the swollen PS-PMA NPs and to study the segmental motions of their polymer chains in the picosecond time scale. The $T_{2}$ time is related to the spectral density function $\left(J\left(\omega_{0}\right)\right.$ at the Larmor frequency $\left.\omega_{0}\right)$ of the spin system, as $1 / T_{2} \propto 3 J(0)+5 J\left(\omega_{0}\right)+2 J\left(2 \omega_{0}\right){ }^{62}$ The spectral density function can be used to calculate the correlation time associated with the local dynamics, in the simplest system, for an isotropic rotation, the relation between the local mobility $\left(\tau_{\mathrm{r}}\right)$ and the spectral density can be expressed by $J\left(\omega_{0}\right)=\tau_{\mathrm{r}} /\left(1-\omega_{0}^{2} \tau_{\mathrm{r}}^{2}\right)$. Similarly, the correlation time of more complex relaxation mechanisms can be extracted from the NMR data. ${ }^{63}$ In every case, an increase in $T_{2}$ is associated with an increase in the local mobility of the segment under study and probes relaxation mechanisms occurring on a time scale similar to the Larmor frequency of the magnet, ${ }^{25}$ $300 \mathrm{MHz}$ in the present study. To characterize the dynamics of PS core and PMA canopy, the $T_{2}$ of the aromatic ring from the PS core and of the methoxy group of the PMA canopy were measured using the Carr-Purcell-Meiboom-Gill pulse sequence (Figure 2). ${ }^{64,65}$ A stretched exponential function

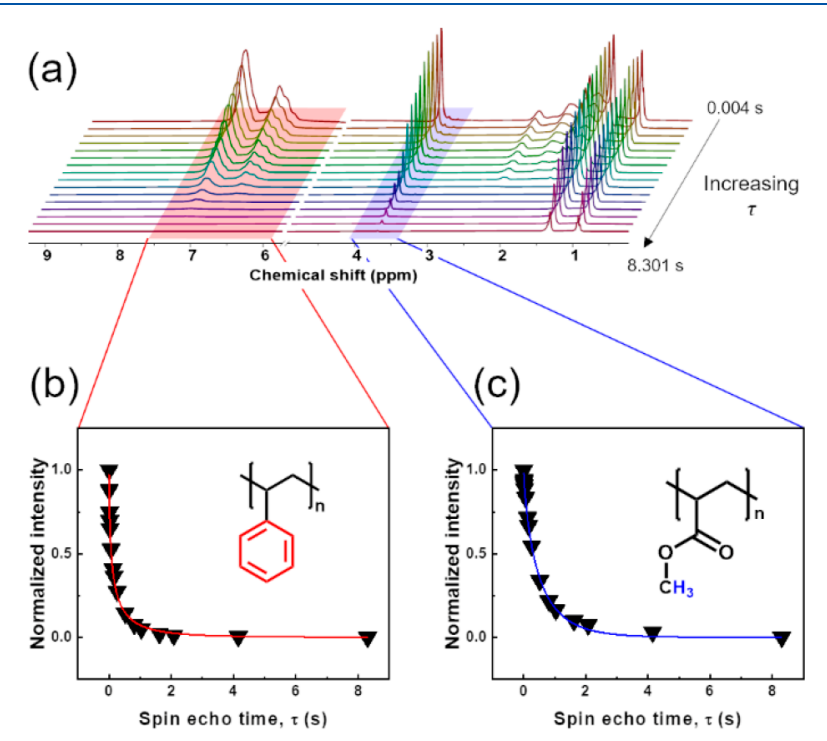

Figure 2. Measurement of the $T_{2}$ in a sample of PS-PMA 4 NPs. (a) ${ }^{1} \mathrm{H}$ NMR spectra of PS-PMA NPs obtained with Carr-PurcellMeiboom-Gill pulse sequence at a Larmor frequency of $300.13 \mathrm{MHz}$ at $298 \mathrm{~K}$. Decay of the signal intensity during the spin-echo extracted from the spectra for (b) aromatic protons of PS and (c) methoxy protons of PMA in the PS-PMA NPs fitted with eq S1.

(eq S3) was used to describe the NMR decay in order to take into account the distribution of confinement states experienced by the polymer chains. The $T_{2}$ values reported for the PS core and of the PMA canopy are the average $T_{2}$ for a given polymer component calculated using eq S4. The relaxation of PS NPs, PS-PMA NPs, and free PMA chains was investigated in mixtures of DCM and cyclohexane and mixtures of DCM and acetone. To understand the effect of the solvent, the NPs were first dispersed in DCM, a good solvent for both PMA and PS, then the addition of a selective nonsolvent, cyclohexane or acetone, was used to tune the solvent quality (Table 1 ).

The quality of the solvent remained below $\chi=0.5$ for both the PS core and the PMA canopy to ensure that the suspensions remained stable during the experiments (Figure 1b). The swelling of the NPs was characterized by their diameter obtained by dynamic light scattering (DLS) in the different solvent mixtures (Figure 3) and normalized to the diameter of the same NPs measure in pure DCM. The solvodynamic diameter $\left(D_{\mathrm{s}}\right)$ of pure PS NPs increased when the mole fraction of cyclohexane increased in the solvent mixture (Figure 3a) and decreased with the addition of 
Table 1. Flory-Huggins Interaction Parameter $\left(\chi_{\text {polymer-solvent }}\right)$ for the Different Polymer-Solvent Pairs Used $^{a}$

\begin{tabular}{lccc} 
& \multicolumn{3}{c}{$\chi_{\text {polymer-solvent }}$} \\
\cline { 2 - 4 } polymer & DCM & acetone & cyclohexane \\
PS & 0.22 & 0.65 & 0.43 \\
PMA & 0.13 & 0.29 & 1.92
\end{tabular}

${ }^{a}$ The $\chi_{\text {polymer-solvent }}$ were calculated from the Hansen solubility parameters of the polymers and solvents (Tables S2 and S3) using eq $\mathrm{S} 1$.
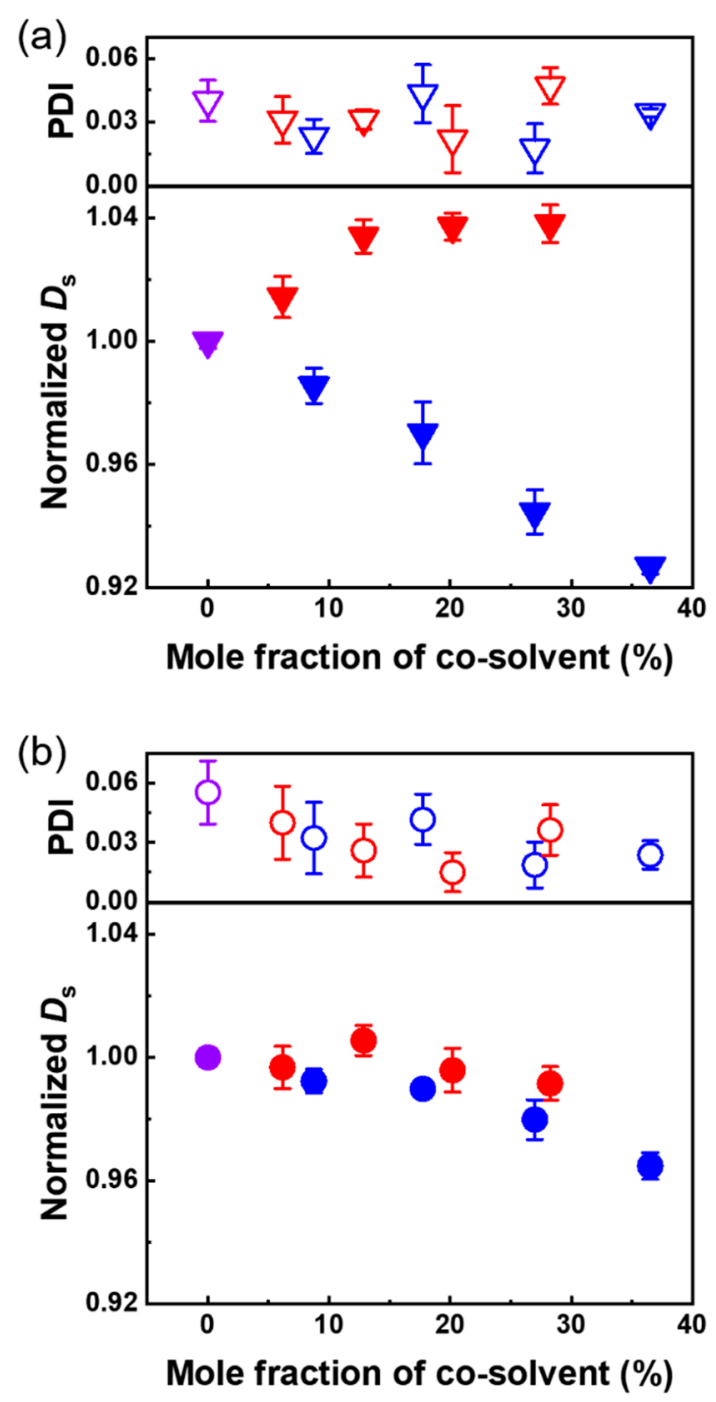

Figure 3. Normalized solvodynamic diameter and size distribution (PDI) of (a) PS NPs ( $\boldsymbol{\nabla}$ ) and (b) PS-PMA $22 \mathrm{k}(\boldsymbol{\bullet})$, measured by light scattering at $298 \mathrm{k}$ in solvent mixtures of DCM (violet) with cyclohexane (red), and with acetone (blue). $D_{\mathrm{s}}$ were normalized by $D_{\mathrm{s}}$ measured in DCM.

acetone. The swelling observed is in agreement with the Flory-Huggins interaction parameter $\left(\chi_{\text {polymer-solvent }}\right)$ of PS in those solvents mixtures (Table S3). For free PMA chains, the radius of gyration of the chain $\left(R_{\mathrm{g}}\right)$ moderately increased with the addition of acetone and decreased with addition of cyclohexane (Figure S2). The mixed system composed of a PS core functionalized with a canopy of end-tethered PMA chains displayed a more intricate behavior (Figure $3 b$ ) due to the simultaneous swelling and deswelling of the different polymer components. PS-PMA $\mathrm{Pk}_{4 \mathrm{k}}$ displayed a similar swelling behavior as the PS NPs (Figure S3). However, as the length of the PMA chains in the PS-PMA NPs increased, the swelling behavior of the PS-PMA particles became sharply distinct from the swelling of pure PS NPs (Figure S3). The effect of the solvent quality on the swelling of the PS-PMA NPs must take into account the simultaneous swelling of the PS core and deswelling of the PMA canopy upon the addition of cyclohexane or the swelling of the PMA canopy accompanied by the deswelling of the PS core upon the addition of acetone.

As the solvent quality changed and the PS core swelled and deswelled, so did the effective grafting density of the chains. Because of the initial size of the NPs and the limited swelling of the PS core, the effective grafting density only moderately changed, ranging from 0.19 chains $\mathrm{nm}^{-2}$ in DCM/cyclohexane to 0.24 chains $\mathrm{nm}^{-2}$ in DCM/acetone (Table S4). Similarly, the swelling and collapse of the PMA canopy could also affect the conformation and dynamics of the system. However, when measuring the variation in the thickness of the polymer canopy with the increase in the degree of polymerization of the PMA chains (Figure S4), the scaling relationship (eq S2) showed that, in every solvent system used, the chains maintained a conformation corresponding to stretched chains in a concentrated polymer brush regime. ${ }^{9}$ Even though the addition of cyclohexane resulted in moderately less stretched chains (Table S5).

Grafting PMA chains to the PS NPs influenced the $T_{2}$ of both PS and PMA in comparison to the free components. The relaxation of the NPs in pure DCM (Figure 4) revealed that the grafting of the PMA chains on the PS core led to an increase in the $T_{2}$ of the PS core. This phenomenon is the consequence of the grafting of mobile PMA chains. At the same time, anchoring the PMA chains to the PS core resulted in a moderate decrease in the $T_{2}$ of the PMA chains due to the substrate effect decreasing the segmental dynamics of the grafted chains in comparison to free PMA chains. ${ }^{66}$ The interplay caused by the tethering of two polymer systems with different intrinsic dynamics is reminiscent of the dynamics of block copolymers, where the dynamics of a slow block can be enhanced by tethering a more rapidly relaxing block and inversely the dynamics of a fast block can be slowed down by the tethering to a slower block. ${ }^{67,68}$ In such situations, the fluctuations the more rapid segment was responsible for the acceleration of the segmental mobility of the slower component.

Changing the quality of the solvent by the addition of cyclohexane, a marginal solvent for PS and a nonsolvent for PMA, changed the local dynamics of both the PS core and the PMA canopy of the PS-PMA NPs (Figure 4). In the case of PS NPs, the $T_{2}$ of the protons of the aromatic ring remained almost constant during the addition of cyclohexane in DCM as the quality of the solvent $\left(\chi_{\text {PS-solvent }}\right)$ changed from 0.22 to 0.09 (Table S3). This observation was consistent with the swelling behavior of PS in DCM/cyclohexane mixture displaying a moderate increase in swelling with the addition of cyclohexane. Under the same conditions, the relaxation of the free PMA chains decreased linearly in keeping with the deswelling of the chains associated with the increase in $\chi_{\text {PMA,solvent }}$ from 0.13 to 0.43. More surprisingly, when the PMA chains were immobilized on the PS core, the $T_{2}$ of both the aromatic protons and the protons of the methoxy group decreased with the addition of cyclohexane. The $T_{2}$ of the methoxy group 
(a)

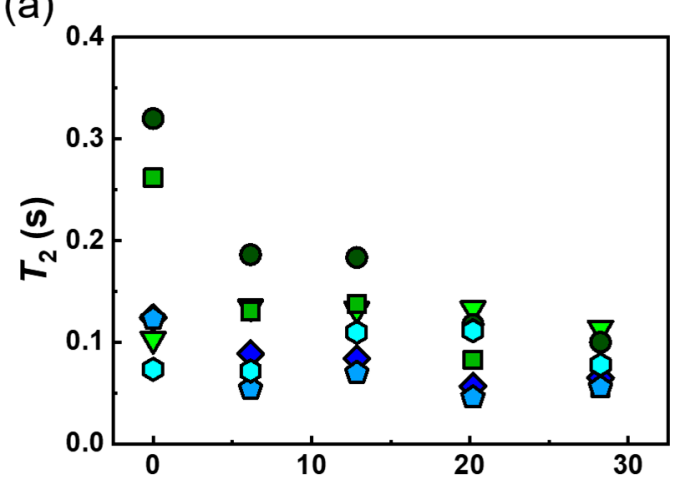

(b) Mole fraction of cyclohexane (\%)

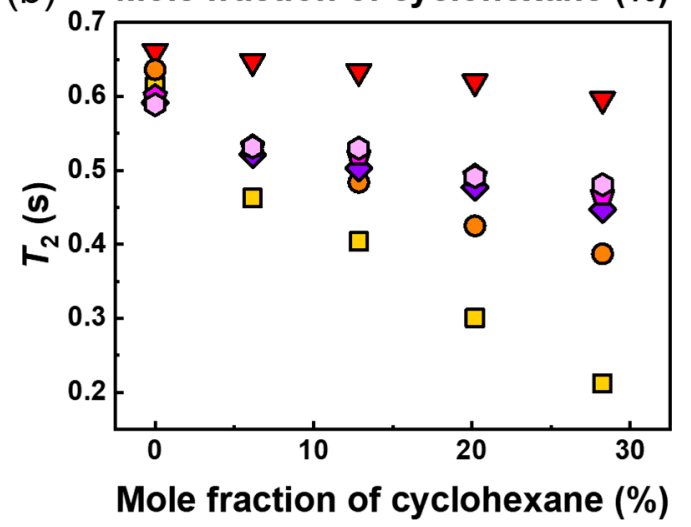

Figure 4. Effect of the addition of selective nonsolvent for the PMA canopy on the local mobility of PS-PMA NPs. $T_{2}$ relaxation of ${ }^{1} \mathrm{H}$ of the (a) aromatic ring of PS of PS NPs (light green triangle), PS$\mathrm{PMA}_{4 \mathrm{k}}$ (green square), $\mathrm{PS}-\mathrm{PMA}_{22 \mathrm{k}}$ (dark green circle), PS-PMA $28 \mathrm{k}$ (blue diamond), $\mathrm{PS}-\mathrm{PMA}_{42 \mathrm{k}}$ (light blue pentagon) and PS-PMA (cyan hexagon) and (b) methoxy group of free $\mathrm{PMA}_{22 \mathrm{k}}$ (red triangle), PS $-\mathrm{PMA}_{4 \mathrm{k}}$ (yellow square), PS- $\mathrm{PMA}_{22 \mathrm{k}}$ (orange circle), PS-PMA (purple diamond), $\mathrm{PS}-\mathrm{PMA}_{42 \mathrm{k}}$ (pink pentagon) and $\mathrm{PS}-\mathrm{PMA}_{52 \mathrm{k}}$ (light pink hexagon) in DCM/cyclohexane mixtures.

decreased even more steeply for PS-PMA NPs than for free PMA chains, and this decrease was more pronounced for the PS-PMA NPs functionalized with short PMA chains. The variation of $T_{2}$ observed with the degree of polymerization of the PMA chains can be ascribed to the different conformational changes occurring in the PMA chains when they are immobilized on the surface of the PS NP, while short chains are forced to adopt an almost fully extended conformation, longer chains, because the radial decrease in the steric crowding in the canopy, can adopt, on average, a more relaxed conformation. More counterintuitively, $T_{2}$ of PS in PS-PMA decreased just like the free PMA since, even, if the solvent condition became better for the PS. This phenomenon can be related to the increased local mobility observed for PS in DCM when PMA chains were grafted to the PS core due to the rapid relaxation of the PMA chains. As the local mobility of the surrounding PMA canopy was reduced, so was the enhancement of the PS local mobility caused by the grafting of the PMA chains. The increase in local mobility observed in pure DCM was no longer present in DCM/cyclohexane solvent mixtures. At high cyclohexane content, the local dynamics of the PS core in PS-PMA NPs became similar to the local dynamics of pure PS NPs since the PMA no longer increased the local mobility of the substrate.
The relaxation behavior of pure PS NPs, PS-PMA, and free PMA chain in DCM/acetone solvent mixture was also studied (Figure 5). Acetone is a good solvent for PMA and a poor

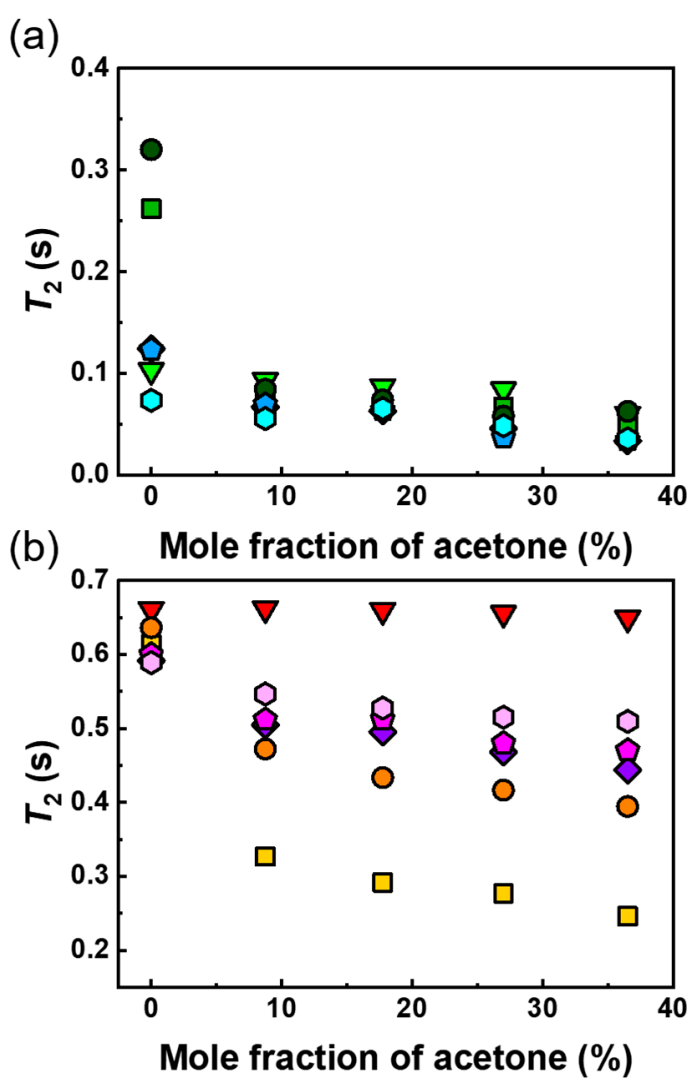

Figure 5. Effect of the addition of selective nonsolvent for the PS core on the local mobility of PS-PMA NPs. $T_{2}$ relaxation of ${ }^{1} \mathrm{H}$ of the (a) aromatic ring of PS of PS NPs (light green triangle), PS-PMA (green square), $\mathrm{PS}-\mathrm{PMA}_{22 \mathrm{k}}$ (dark green circle), $\mathrm{PS}-\mathrm{PMA}_{28 \mathrm{k}}$ (blue diamond), $\mathrm{PS}-\mathrm{PMA}_{42 \mathrm{k}}$ (light blue pentagon), and PS-PMA $52 \mathrm{k}$ (cyan hexagon) and (b) methoxy group of free $\mathrm{PMA}_{22 \mathrm{k}}$ (red triangle), PS$\mathrm{PMA}_{4 \mathrm{k}}$ (yellow square), PS-PMA ${ }_{22 \mathrm{k}}$ (orange circle), PS-PMA $28 \mathrm{k}$ (purple diamond), PS- $\mathrm{PMA}_{42 \mathrm{k}}$ (pink pentagon), and PS-PMA (light pink hexagon) in DCM/acetone mixtures.

solvent for PS. Upon the addition of acetone to DCM the $T_{2}$ of free PMA chains remained constant as the solvent quality $\chi_{\text {PMA-solvent }}$ changed from 0.13 to 0.16 . Simultaneously, the $T_{2}$ of pure PS NPs moderately decreased when the mole fraction of acetone increased in the solvent mixture due to the nonsolvency of PS in acetone with $\chi_{\mathrm{PS} \text {,solvent }}$ increasing from 0.22 to 0.35 . The decrease in $\chi_{\mathrm{PS} \text {,solvent }}$ was also associated with the deswelling of the PS NPs (Figure 3). After the addition of acetone, the $T_{2}$ of the tethered PMA chains significantly decreased, in keeping with the decrease of the local mobility of the PS core, this phenomenon was more pronounced for short PMA chains and systematically reduced as the length of the end-tethered PMA chains increased. Therefore, the local mobility of short chains was more susceptible to be influenced by the dynamics of the substrate, especially when the substrate was soft and deformable.

The variation of the $T_{2}$ of PS and PMA as a function of $\chi_{\text {polymer-solvent }}$ of the polymers in the different solvent mixtures (Figure 6) emphasized the existence of an interplay between the dynamics of the PMA canopy and the dynamics of the PS core. Pure PS NPs and free PMA chains both showed the 
(a)

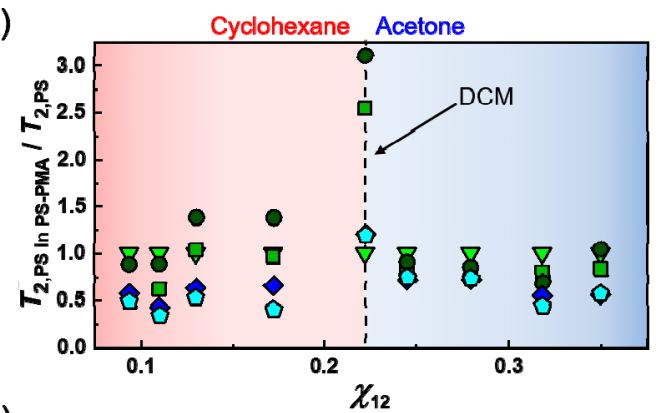

(b)

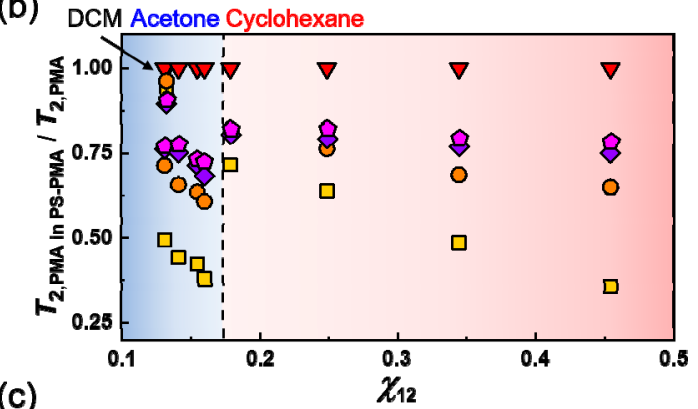

(c)

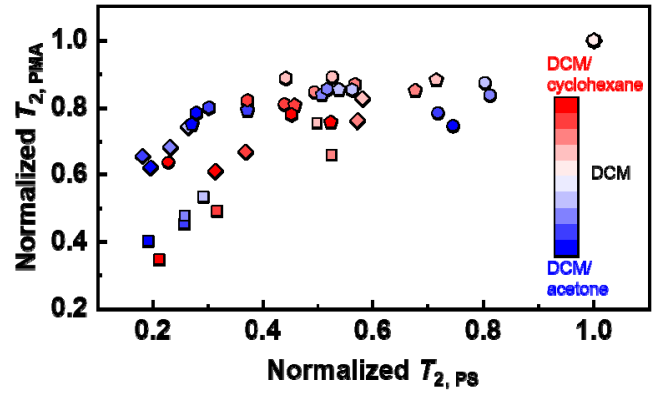

Figure 6. Normalized $T_{2}$ relaxation time of ${ }^{1} \mathrm{H}$ of the (a) aromatic ring of PS of PS NPs (light green triangle), PS- $\mathrm{PMA}_{4 \mathrm{k}}$ (green square), $P S-\mathrm{PMA}_{22 \mathrm{k}}$ (dark green circle), $\mathrm{PS}-\mathrm{PMA}_{28 \mathrm{k}}$ (blue diamond), and PS-PMA $42 \mathrm{k}$ (light blue pentagon) and (b) methoxy group of free $\mathrm{PMA}_{22 \mathrm{k}}$ (red triangle), PS-PMA $4 \mathrm{k}$ (yellow square), PS$\mathrm{PMA}_{22 \mathrm{k}}$ (orange circle), PS- $\mathrm{PMA}_{28 \mathrm{k}}$ (purple diamond), and PS$\mathrm{PMA}_{42 \mathrm{k}}$ (pink pentagon) as a function of $\chi_{12}$ of PS and PMA. $T_{2}$ normalized to the $T_{2}$ of the pure PS NPs or free PMA chains in the same solvent composition. (c) Relation between the variation of the $T_{2}$ relaxation time of PS and PMA in PS-PMA NPs in solvent mixtures normalized to the $T_{2}$ observed for PS-PMA NPs in pure DCM.

expected overall linear decrease in $T_{2}$ when the $\chi_{\text {PS-solvent }}$ varies from a good solvent to a marginal solvent (Figure S6). However, the local mobility of the grafted core-canopy system was more complex because of the synergetic effect of the cosolvent addition. The normalized $T_{2}$ of PS in PS-PMA NPs increased as the solvent quality decreased $\left(\chi_{\mathrm{PS}-\text { solvent }}\right.$ increasing from 0.09 to 0.22 ) and then followed the trend of pure PS NPs and decreased with a further increase of $\chi_{\text {PS-solvent }}$. This change in behavior occurred when the cosolvent used to tune the solvent quality changed from cyclohexane to acetone and was ascribed to the interplay between the local mobility of the core and the local mobility of the PMA canopy. Initially, the addition of acetone decreased the solvent quality for the PS core but had a limited influence of the solvent quality for the PMA canopy (Figure S7). Consequently, the local mobility of the PS core followed the same trend as the local mobility of the unfunctionalized PS NPs since there was no other effects. However, the addition of cyclohexane to the DCM suspension was accompanied by an increase in the solvent quality for the PS core and a decrease in the solvent quality for the PMA canopy, and the behavior of the PS core deviated from the behavior of the unfunctionalized PS NPs, because the local mobility of the PS core was not only influenced by $\chi_{\text {PS-solvent }}$ but also by the behavior of the PMA canopy. The grafting of the PMA canopy increased the local mobility of the PS core, but this effect was observable only when the PMA canopy was in a good solvent and sufficiently mobile.

The variation of the $T_{2}$ of the PMA canopy on the PS-PMA NPs was characterized by a discontinuity when the solvent mixture was changed from DCM/acetone to DCM/cyclohexane mixture. First, $T_{2}$ decreased as $\chi_{\text {PMA-solvent }}$ increased from 0.13 to 0.16 with the addition of acetone, then increased drastically when the solvent mixture was changed from $36 \mathrm{~mol}$ $\%$ of acetone to $6 \mathrm{~mol} \%$ of cyclohexane in DCM, although $\chi_{\text {PMA-solvent }}$ only changed from 0.16 and 0.18 . Then, with the further addition of cyclohexane, the $T_{2}$ of the PMA canopy decreased again with the increases of $\chi_{\text {PMA-solvent }}$. Albeit the change in solvent quality experienced by the PMA chains was limited when switching the cosolvent from acetone to cyclohexane, the $T_{2}$ of the PMA canopy was simultaneously influenced by the large variation in $\chi_{\text {PS-solvent }}$ from 0.35 to 0.17 , sustained by the PS (Figure S5) resulting in a decrease in the local mobility of the PS core.

Consequently, the effect of $\chi_{\text {polymer-solvent }}$ for one single component of the PS-PMA system cannot solely be used to explain the variations observed in the relaxation dynamics. The simultaneous impact of the change of the solvent quality on both PS and PMA should be considered as well as the interplay between the local dynamics of the core and of the canopy. When the solvent mixture was changed from $36 \mathrm{~mol} \%$ acetone in DCM to $6 \mathrm{~mol} \%$ cyclohexane in DCM, the $\chi_{\text {PMA-solvent }}$ changed from 0.16 to 0.18 , but the $\chi_{\text {PS-solvent }}$ changed from 0.35 to 0.17 . When comparing those two solvent mixtures, the local mobility of the naked PS NPs increased 3-folds going from acetone to cyclohexane. Thus, the substrate of the PMA brushes had a different dynamics going from $36 \mathrm{~mol} \%$ of acetone to $6 \mathrm{~mol} \%$ of cyclohexane and resulted in the discontinuous behavior observed for the $T_{2}$ of the PMA canopy. As the local mobility of the PS increased, the substrate effect limiting the local mobility of the grafted chain was less prominent, and the $T_{2}$ of the PMA chains increased. The addition of more cyclohexane led to a steady decrease in the $T_{2}$ of the PMA canopy in keeping with the behavior of free PMA chains. An additional evidence of the interplay between the dynamics of the core and the canopy was the effect of the degree of polymerization of the PMA chains on local mobility of the system. The impact of the solvent quality on the local mobility of PMA chains was more pronounced for the shorter PMA chains, for which the local mobility of the canopy was more strongly dependent on the local mobility of the PS substrate.

The interplay between the PS core and the PMA canopy could only be understood by concurrently considering the effect of $\chi_{\text {PS-solvent }}$ and $\chi_{\text {PMA-solvent }}$ on the $T_{2}$ of both PS and PMA, (Figure S8). The solvent quality had a definite impact on the $T_{2}$ of both the PS and PMA segments. However, the $T_{2}$ of the PS was also influenced by the relaxation of the PMA segments, and inversely the relaxation of the PMA segments was affected by the local mobility of the PS core (Figure 6c). In the PS-PMA core-canopy system, the reduction of the relaxation time of the core was systematically accompanied 
by a decrease of the relaxation time of the canopy irrespectively of the chain length. Inversely, a reduction of the $T_{2}$ of the canopy led to a reduction of the $T_{2}$ of the core. Consequently, only a common good solvent resulted in fast local mobility for either PS or PMA in the coupled system. The addition of a selective nonsolvent resulted in the decrease in the relaxation of both the core and the canopy irrespectively if the nonsolvent was selective for the core or the canopy.

\section{CONCLUSIONS}

In conclusion, the study of the relaxation dynamics of soft swollen gel nanoparticles functionalized with polymer chains was investigated by NMR relaxometry. The effect of the solvent quality on the swelling behavior and the relaxation dynamics of PS core and PMA canopy of PS-PMA hairy soft NPs were investigated using solvent mixtures with tunable and selective solvent quality favoring either the PS core or the PMA canopy. The results suggest a strong interplay between the dynamics of the core and the dynamics of the canopy of endtethered chains.

In a single polymer system, either pure PS NPs of free PMA chains, the $T_{2}$ of the polymer decreased with decreasing $\chi_{\text {polymer-solvent }}$. However, in the case of PS NPs functionalized with a canopy of end-tethered PMA chains, the effect of the solvent quality on the $T_{2}$ of the polymer was more complex due to the interplay between the mobility of the PS core and of the PMA canopy. Generally, the functionalization of the PS core with a canopy of swollen and mobile PMA chains increased the $T_{2}$ of the PS in comparison to unfunctionalized PS NPs. As expected, the addition of a selective nonsolvent for the PMA canopy reduced the solvent quality for the PMA canopy, and a reduction in the local mobility of the PMA chains was observed. However, this reduction in the local mobility of the PMA canopy triggered a reduction in the local mobility of the PS core, although the solvent quality for the PS core increased in that solvent mixture. Similarly, as the local mobility of the PS decreased with the addition of a selective nonsolvent, the $T_{2}$ of the PMA canopy also reduced due to a stronger immobilization by the PS core.

Selectively decreasing the solvent quality for one of the polymer components, either the PS core or the PMA canopy, resulted in a decrease in the mobility of both polymer components. When a polymer system with a complex architecture, such as the core-canopy PS-PMA NPs under study, are used, the synergetic effect between composition, architecture, and environment need to be considered during their processing.

\section{ASSOCIATED CONTENT}

\section{S1 Supporting Information}

The Supporting Information is available free of charge at https://pubs.acs.org/doi/10.1021/acs.macromol.0c00346.

Detailed procedure for the synthesis of the nanoparticles, the calculation of the Flory-Huggins interaction parameters, and additional information about the characterization of the NPs by DLS, TEM, and NMR spectroscopy. (PDF)

\section{AUTHOR INFORMATION}

\section{Corresponding Author}

Héloïse Thérien-Aubin - Max Planck Institute for Polymer Research, 55128 Mainz, Germany; 이이이.org/0000-00034567-516X; Email: therien@mpip-mainz.mpg.de

\section{Author}

Young-Gon Kim - Max Planck Institute for Polymer Research, 55128 Mainz, Germany

Complete contact information is available at:

https://pubs.acs.org/10.1021/acs.macromol.0c00346

\section{Notes}

The authors declare no competing financial interest.

\section{ACKNOWLEDGMENTS}

The authors acknowledge Prof. Dr. Kartharina Landfester for fruitful discussions and support. The authors are grateful for the financial support of the Max Planck Center for Complex Fluid Dynamics. H.T.-A. is grateful to the Alexander von Humboldt Foundation for financial support. The authors gratefully thank Ms. Christine Rosenauer for light scattering measurement. The authors are thankful to Ms. Katrin Kirchhoff for transmission electron microscopy measurement.

\section{REFERENCES}

(1) Milner, S. T. Polymer Brushes. Science 1991, 251 (4996), 905914.

(2) Pincus, P. Colloid Stablization with Grafted Polyelectrolytes. Macromolecules 1991, 24 (10), 2912-2919.

(3) Synytska, A.; Svetushkina, E.; Puretskiy, N.; Stoychev, G.; Berger, S.; Ionov, L.; Bellmann, C.; Eichhorn, K. J.; Stamm, M. Biocompatible Polymeric Materials with Switchable Adhesion Properties. Soft Matter 2010, 6 (23), 5907-5914.

(4) Song, J. B.; Zhou, J. J.; Duan, H. W. Self-Assembled Plasmonic Vesicles of SERS-Encoded Amphiphilic Gold Nanoparticles for Cancer Cell Targeting and Traceable Intracellular Drug Delivery. J. Am. Chem. Soc. 2012, 134 (32), 13458-13469.

(5) Zhan, X. L.; Yan, Y. D.; Zhang, Q. H.; Chen, F. Q. A Novel Superhydrophobic Hybrid Nanocomposite Material Prepared by Surface-Initiated AGET ATRP and its Anti-Icing Properties. J. Mater. Chem. A 2014, 2 (24), 9390-9399.

(6) Zoppe, J. O.; Ataman, N. C.; Mocny, P.; Wang, J.; Moraes, J.; Klok, H.-A. Surface-Initiated Controlled Radical Polymerization: State-of-the-Art, Opportunities, and Challenges in Surface and Interface Engineering with Polymer Brushes. Chem. Rev. 2017, 117 (3), 1105-1318.

(7) Akcora, P.; Liu, H.; Kumar, S. K.; Moll, J.; Li, Y.; Benicewicz, B. C.; Schadler, L. S.; Acehan, D.; Panagiotopoulos, A. Z.; Pryamitsyn, V.; Ganesan, V.; Ilavsky, J.; Thiyagarajan, P.; Colby, R. H.; Douglas, J. F. Anisotropic Self-Assembly of Spherical Polymer-Grafted Nanoparticles. Nat. Mater. 2009, 8 (4), 354-U121.

(8) Sunday, D.; Ilavsky, J.; Green, D. L. A Phase Diagram for Polymer-Grafted Nanoparticles in Homopolymer Matrices. Macromolecules 2012, 45 (9), 4007-4011.

(9) Hore, M. J. A. Polymers on nanoparticles: structure \& dynamics. Soft Matter 2019, 15 (6), 1120-1134.

(10) Srivastava, S.; Kandar, A. K.; Basu, J. K.; Mukhopadhyay, M. K.; Lurio, L. B.; Narayanan, S.; Sinha, S. K. Complex dynamics in polymer nanocomposites. Phys. Rev. E 2009, 79 (2), 021408.

(11) Wei, Y.; Xu, Y.; Faraone, A.; Hore, M. J. A. Local Structure and Relaxation Dynamics in the Brush of Polymer-Grafted Silica Nanoparticles. ACS Macro Lett. 2018, 7 (6), 699-704.

(12) Jiang, N.; Endoh, M. K.; Koga, T.; Masui, T.; Kishimoto, H.; Nagao, M.; Satija, S. K.; Taniguchi, T. Nanostructures and Dynamics 
of Macromolecules Bound to Attractive Filler Surfaces. ACS Macro Lett. 2015, 4 (8), 838-842.

(13) Weaver, J. V. M.; Tang, Y.; Liu, S.; Iddon, P. D.; Grigg, R.; Billingham, N. C.; Armes, S. P.; Hunter, R.; Rannard, S. P. Preparation of Shell Cross-Linked Micelles by Polyelectrolyte Complexation. Angew. Chem., Int. Ed. 2004, 43 (11), 1389-1392.

(14) Posel, Z.; Posocco, P. Tuning the Properties of Nanogel Surfaces by Grafting Charged Alkylamine Brushes. Nanomaterials 2019, 9 (11), 1514.

(15) Wichaita, W.; Kim, Y. G.; Tangboriboonrat, P.; Thérien-Aubin, $\mathrm{H}$. Polymer-functionalized polymer nanoparticles and their behaviour in suspensions. Polym. Chem. 2020, 11 (12), 2119-2128.

(16) Daoud, M.; Cotton, J. P. Star Shaped Polymers: A Model for the Conformation and Its Concentration Dependence. J. Phys. (Paris) 1982, 43 (3), 531-538.

(17) Bachhar, N.; Kumaraswamy, G.; Kumar, S. K. Core-Size Dispersity Dominates the Self-Assembly of Polymer-Grafted Nanoparticles in Solution. Macromolecules 2019, 52 (13), 4888-4894.

(18) Kim, S. Y.; Meyer, H. W.; Saalwächter, K.; Zukoski, C. F. Polymer Dynamics in PEG-Silica Nanocomposites: Effects of Polymer Molecular Weight, Temperature and Solvent Dilution. Macromolecules 2012, 45 (10), 4225-4237.

(19) Martin, T. B.; Mongcopa, K. I. S.; Ashkar, R.; Butler, P.; Krishnamoorti, R.; Jayaraman, A. Wetting-Dewetting and Dispersion-Aggregation Transitions Are Distinct for Polymer Grafted Nanoparticles in Chemically Dissimilar Polymer Matrix. J. Am. Chem. Soc. 2015, 137 (33), 10624-10631.

(20) Yang, S.; Akcora, P. Deformation of Chemically Heterogeneous Interfacial Layers of Polymer Nanocomposites. ACS Macro Lett. 2019, 8 (12), 1635-1641.

(21) Kim, S. A.; Mangal, R.; Archer, L. A. Relaxation Dynamics of Nanoparticle-Tethered Polymer Chains. Macromolecules 2015, 48, 6280.

(22) Holt, A. P.; Bocharova, V.; Cheng, S.; Kisliuk, A. M.; White, B. T.; Saito, T.; Uhrig, D.; Mahalik, J. P.; Kumar, R.; Imel, A. E.; Etampawala, T.; Martin, H.; Sikes, N.; Sumpter, B. G.; Dadmun, M. D.; Sokolov, A. P. Controlling Interfacial Dynamics: Covalent Bonding versus Physical Adsorption in Polymer Nanocomposites. ACS Nano 2016, 10 (7), 6843-6852.

(23) Poling-Skutvik, R.; Olafson, K. N.; Narayanan, S.; Stingaciu, L.; Faraone, A.; Conrad, J. C.; Krishnamoorti, R. Confined Dynamics of Grafted Polymer Chains in Solutions of Linear Polymer. Macromolecules 2017, 50 (18), 7372-7379.

(24) Richter, D.; Kruteva, M. Polymer Dynamics under Confinement. Soft Matter 2019, 15 (37), 7316-7349.

(25) McLeish, T. C. B. Tube theory of entangled polymer dynamics. Adv. Phys. 2002, 51 (6), 1379-1527.

(26) Heatley, F. Nuclear Magnetic-Relaxation of Synthetic-Polymers in Dilute-Solution. Prog. Nucl. Magn. Reson. Spectrosc. 1979, 13, 4785.

(27) Dais, P.; Spyros, A. ${ }^{13} \mathrm{C}$ Nuclear magnetic relaxation and local dynamics of synthetic polymers in dilute solution and in the bulk state. Prog. Nucl. Magn. Reson. Spectrosc. 1995, 27 (5), 555-633.

(28) Klein, P. G.; Ries, M. E. The dynamics and physical structure of polymers above the glass transition-transverse relaxation studies of linear chains, star polymers and networks. Prog. Nucl. Magn. Reson. Spectrosc. 2003, 42 (1), 31-52.

(29) Palmer, A. G. NMR characterization of the dynamics of biomacromolecules. Chem. Rev. 2004, 104 (8), 3623-3640.

(30) Pinto, L. F.; Correa, J.; Martin-Pastor, M.; Riguera, R.; Fernandez-Megia, E. The Dynamics of Dendrimers by NMR Relaxation: Interpretation Pitfalls. J. Am. Chem. Soc. 2013, 135 (5), 1972-1977.

(31) Charlier, C.; Khan, S. N.; Marquardsen, T.; Pelupessy, P.; Reiss, V.; Sakellariou, D.; Bodenhausen, G.; Engelke, F.; Ferrage, F. Nanosecond Time Scale Motions in Proteins Revealed by HighResolution NMR Relaxometry. J. Am. Chem. Soc. 2013, 135 (49), $18665-18672$.
(32) Ashkar, R. Selective dynamics in polymeric materials: Insights from quasi-elastic neutron scattering spectroscopy. J. Appl. Phys. 2020, 127 (15), 151101.

(33) Cendoya, I.; Alegria, A.; Alberdi, J. M.; Colmenero, J.; Grimm, H.; Richter, D.; Frick, B. Effect of Blending on the PVME Dynamics. A Dielectric, NMR, and QENS Investigation. Macromolecules 1999, 32 (12), 4065-4078.

(34) Derakhshan, M.; Ansarian, H. R.; Rahman, M. M.; Sakurai, T.; Takafuji, M.; Ihara, H. A new method for evaluation of the mobility of silica-grafted alkyl chains by suspension-state 1H NMR. Can. J. Chem. 2005, 83 (10), 1792-1798.

(35) Friedrichs, C.; Emmerling, S.; Kircher, G.; Graf, R.; Wolfgang Spiess, H. Glass transition of poly(ethylmethacrylate) admixed and bound to nanoparticles. J. Chem. Phys. 2013, 138 (12), 12 A503.

(36) Spiess, H. W. 50th Anniversary Perspective: The Importance of NMR Spectroscopy to Macromolecular Science. Macromolecules 2017, 50 (5), 1761-1777.

(37) Schweizerhof, S.; Demco, D. E.; Mourran, A.; Keul, H.; Fechete, R.; Möller, M. Temperature-Induced Phase Transition Characterization of Responsive Polymer Brushes Grafted onto Nanoparticles. Macromol. Chem. Phys. 2017, 218 (6), 1600495.

(38) Dimitrov, D. I.; Milchev, A.; Binder, K. Polymer brushes in solvents of variable quality: Molecular dynamics simulations using explicit solvent. J. Chem. Phys. 2007, 127 (8), 084905.

(39) Borisov, O. V.; Birshtein, T. M.; Zhulina, E. B. Collapse of Grafted Polyelectrolyte Layer. J. Phys. II 1991, 1 (5), 521-526.

(40) Nomura, A.; Okayasu, K.; Ohno, K.; Fukuda, T.; Tsujii, Y. Lubrication Mechanism of Concentrated Polymer Brushes in Solvents: Effect of Solvent Quality and Thereby Swelling State. Macromolecules 2011, 44 (12), 5013-5019.

(41) Grest, G. S.; Murat, M. Structure of Grafted Polymeric Brushes in Solvents of Varying Quality - A Molecular-Dynamic Study. Macromolecules 1993, 26 (12), 3108-3117.

(42) LoVerso, F.; Egorov, S. A.; Binder, K. Interaction Between Polymer Brush-Coated Spherical Nanoparticles: Effect of Solvent Quality. Macromolecules 2012, 45 (21), 8892-8902.

(43) Choueiri, R. M.; Galati, E.; Thérien-Aubin, H.; Klinkova, A.; Larin, E. M.; Querejeta-Fernández, A.; Han, L.; Xin, H. L.; Gang, O.; Zhulina, E. B.; Rubinstein, M.; Kumacheva, E. Surface patterning of nanoparticles with polymer patches. Nature 2016, 538 (7623), 7983.

(44) Zhang, Q.; Liao, Y.; He, L. P.; Bu, W. F. Spherical Polymer Brushes in Solvents of Variable Quality: An Experimental Insight by TEM Imaging. Langmuir 2013, 29 (13), 4181-4186.

(45) Klinkova, A.; Therien-Aubin, H.; Choueiri, R. M.; Rubinstein, M.; Kumacheva, E. Colloidal analogs of molecular chain stoppers. Proc. Natl. Acad. Sci. U. S. A. 2013, 110 (47), 18775-18779.

(46) Zhang, J.; Pelton, R. Poly(N-isopropylacrylamide) Microgels at the Air-Water Interface. Langmuir 1999, 15 (23), 8032-8036.

(47) Senff, H.; Richtering, W. Temperature Sensitive Microgel Suspensions: Colloidal Phase Behavior and Rheology of Soft Spheres. J. Chem. Phys. 1999, 111 (4), 1705-1711.

(48) Gao, J.; Hu, Z. B. Optical Properties of N-isopropylacrylamide Microgel Spheres in Water. Langmuir 2002, 18 (4), 1360-1367.

(49) Cai, T.; Marquez, M.; Hu, Z. Monodisperse Thermoresponsive Microgels of Poly(ethylene glycol) Analogue-Based Biopolymers. Langmuir 2007, 23 (17), 8663-8666.

(50) Xun, W. W.; Richtering, W. Dilution Leading to Viscosity Increase based on the Cononsolvency Effect of Temperature-Sensitive Microgel Suspensions. Colloids Surf., A 2015, 484, 377-385.

(51) Scherzinger, C.; Lindner, P.; Keerl, M.; Richtering, W. Cononsolvency of Poly(N,N-diethylacrylamide) (PDEAAM) and Poly(N-isopropylacrylamide) (PNIPAM) Based Microgels in Water/Methanol Mixtures: Copolymer vs Core-Shell Microgel. Macromolecules 2010, 43 (16), 6829-6833.

(52) Scherzinger, C.; Holderer, O.; Richter, D.; Richtering, W. Polymer Dynamics in Responsive Microgels: Influence of Cononsolvency and Microgel Architecture. Phys. Chem. Chem. Phys. 2012, 14 (8), 2762-2768. 
(53) Chen, L.; Peng, Z. P.; Zeng, Z. P.; She, Y. Q.; Wei, J. C.; Chen, Y. W. Hairy Polymeric Nanocapsules with $\mathrm{pH}$-Responsive Shell and Thermoresponsive Brushes: Tunable Permeability for Controlled Release of Water-Soluble Drugs. J. Polym. Sci., Part A: Polym. Chem. 2014, 52 (15), 2202-2216.

(54) Berndt, I.; Pedersen, J. S.; Richtering, W. TemperatureSensitive Core-Shell Microgel Particles with Dense Shell. Angew. Chem., Int. Ed. 2006, 45 (11), 1737-1741.

(55) Kim, Y.-G.; Wagner, M.; Thérien-Aubin, H. Dynamics of Soft and Hairy Polymer Nanoparticles in a Suspension by NMR Relaxation. Macromolecules 2020, 53, 844.

(56) Landfester, K. Miniemulsion Polymerization and the Structure of Polymer and Hybrid Nanoparticles. Angew. Chem., Int. Ed. 2009, 48 (25), 4488-4507.

(57) Schreiber, E.; Ziener, U.; Manzke, A.; Plettl, A.; Ziemann, P.; Landfester, K. Preparation of Narrowly Size Distributed MetalContaining Polymer Latexes by Miniemulsion and Other Emulsion Techniques: Applications for Nanolithography. Chem. Mater. 2009, 21 (8), 1750-1760.

(58) Min, K.; Gao, H. F.; Matyjaszewski, K. Use of Ascorbic Acid as Reducing Agent for Synthesis of Well-defined Polymers by ARGET ATRP. Macromolecules 2007, 40 (6), 1789-1791.

(59) Tanaka, N.; Matsukawa, S.; Kurosu, H.; Ando, I. A Study on Dynamics of Water in Crosslinked Poly(N-isopropylacrylamide) Gel by NMR Spectroscopy. Polymer 1998, 39 (20), 4703-4706.

(60) Pietrasik, J.; Sumerlin, B. S.; Lee, H. I.; Gil, R. R.; Matyjaszewski, K. Structural Mobility of Molecular Bottle-Brushes Investigated by NMR Relaxation Dynamics. Polymer 2007, 48 (2), 496-501.

(61) Besghini, D.; Mauri, M.; Simonutti, R. Time Domain NMR in Polymer Science: From the Laboratory to the Industry. Appl. Sci. 2019, 9 (9), 1801.

(62) Bloembergen, N.; Purcell, E. M.; Pound, R. V. Relaxation Effects in Nuclear Magnetic Resonance Absorption. Phys. Rev. 1948, 73 (7), 679-712.

(63) Lipari, G.; Szabo, A. Model-free approach to the interpretation of nuclear magnetic resonance relaxation in macromolecules. 1. Theory and range of validity. J. Am. Chem. Soc. 1982, 104 (17), $4546-4559$.

(64) Carr, H. Y.; Purcell, E. M. Effects of Diffusion on Free Precesion in Nuclear Magnetic Resonance Experiments. Phys. Rev. 1954, 94 (3), 630-638.

(65) Meiboom, S.; Gill, D. Modified Spin-Echo Method for Measuring Nuclear Relaxation Times. Rev. Sci. Instrum. 1958, 29 (8), 688-691.

(66) Savin, D. A.; Pyun, J.; Patterson, G. D.; Kowalewski, T.; Matyjaszewski, K. Synthesis and Characterization of Silica-graftPolystyrene Hybrid Nanoparticles: Effect of Constraint on the GlassTransition Temperature of Spherical Polymer Brushes. J. Polym. Sci., Part B: Polym. Phys. 2002, 40 (23), 2667-2676.

(67) Lund, R.; Willner, L.; Alegría, A.; Colmenero, J.; Richter, D. Self-Concentration and Interfacial Fluctuation Effects on the Local Segmental Dynamics of Nanostructured Diblock Copolymer Melts. Macromolecules 2008, 41 (3), 511-514.

(68) Alegria, A.; Lund, R.; Barroso-Bujans, F.; Arbe, A.; Colmenero, J. Component dynamics in nanostructured PI-PDMS diblock copolymers with PI segregated in lamellas, cylinders, and spheres. Colloid Polym. Sci. 2014, 292 (8), 1863-1876. 